\title{
Synthesis, structure and spectroscopic properties of cobalt(III) complexes with 1- benzoyl-(3,3-disubstituted)thiourea
}

\begin{abstract}
Reactions of $\mathrm{Co}$ (II) acetate with 1-benzoyl-(3-methyl-3-R)thiourea ( $\mathrm{R}=$ ethyl, propyl, cyclohexyl and phenyl) and 1-benzoyl-(3-ethyl-3-benzyl)thiourea ligands, herein refer to as 1-benzoyl-(3,3-disubstituted)thiourea, derived from secondary alkyl- and arylamines gave [tris(1-benzoyl-(3,3-disubstituted)thiourea)cobalt(III)] complexes. The neutral cobalt(III) complexes were afforded through a one-pot reaction between a deprotonated 1-benzoyl-(3,3disubstituted)thiourea ligands with cobalt(II) acetate with a 1:3 ratio in methanol. These complexes were characterized on the basis of elemental analysis, mass spectrometry, magnetic susceptibility measurement, X-ray crystallography and spectroscopic techniques namely infrared, UVï Vis and nuclear magnetic resonance. Based on the magnetic susceptibility and spectroscopic data as well as X-ray crystallographic structure of the octahedron [tris(1-benzoyl-(3-methyl-3-phenyl)thiourea)cobalt(III)] complex, it can be concluded that the ligands behave as bidentate $\mathrm{O}, \mathrm{S}$ chelate.
\end{abstract}

Keyword: Benzoylthiourea; Cobalt(III); X-ray crystallography; Bidentate-chelate; Thiourea; One-pot-reaction 